\title{
In Vitro Evaluation of Prebiotics on Adherence of Lactobacilli
}

\section{Krausova G*, Hyrslova I, Jakubec M and Hynstova I}

Dairy Research Institute, Ke Dvoru 12a, 16000 Prague, Czech Republic

\begin{abstract}
The ability to adhere to intestinal epithelia is a crucial probiotic property since the retention of bacteria in the large intestine is important for probiotic function. Previously, we have shown that the presence of prebiotics decreases the adherence in several prebiotic- probiotic combinations. The objective of this study was to test the effect of three commercially available prebiotics: Orafti GR, Orafti P95, and Orafti Synergy on the adherence of five Lactobacillus strains (Lactobacillus delbrueckii subsp. bulgaricus CCDM 66, Lactobacillus casei subsp. paracasei PE1TB-P, Lactobacillus fermentum RL25, Lactobacillus animalis CCDM 382, and Lactobacillus gasseri PHM-7E1). Adherence was tested using microtiter plates and was evaluated as the percentage of fluorescently labelled bacteria present in the wells after three washes. Adherence was evaluated in both uncoated and mucin-coated polystyrene plates. In accordance with our previous results, the majority of probiotic and prebiotic interactions resulted in at least a 10-fold decrease in adherence. Only one strain, L. gasseri PHM-7E1, exhibited increased adherence to mucin (from $5 \%$ to $8 \%$ and from $5 \%$ to $9 \%$ ) after the addition of the prebiotics Orafti P95 and GR, respectively. These two combinations appear suitable for further synbiotic testing.
\end{abstract}

Keywords: Adhesion; Adherence; Lactobacilli; Prebiotics

\section{Introduction}

The ability to adhere to the intestinal epithelium is one of the most important properties of potential probiotic microorganisms [1]. The capacity of prebiotics to enhance probiotic properties is well described; prebiotics are known to serve as an energy source for bacteria that enhance their growth. Prebiotics are defined as non-digestible compounds, providing beneficial physiological effects for the host by selectively stimulating the growth or activity of a limited number of bacterial species present in the colon [2]. These compounds include oligosaccharides (usually fructo- and galacto-oligosaccharides) that can be fermented to organic acids by colonic microbiota $[3,4]$ and are therefore considered selective substrates for probiotics [5]. The therapeutic potential of prebiotic oligosaccharides as well as their beneficial effect on bacterial growth is well established. However, little is known regarding the impact of prebiotics (or saccharides in general) on the adherence of beneficial bacteria, such as bifidobacteria or lactobacilli. Previous studies have mainly focused on the influence of prebiotics on the adherence of pathogens, such as Helicobacter pylori, Campylobacter jejuni, Clostridium difficile and others. As demonstrated, prebiotics can inhibit the adhesion of pathogenic bacteria [4,6,7]; infectious bacteria bind to prebiotics instead of epithelial receptor sites [4], thus blocking the attachment of microorganisms and their toxins to host cell surfaces. This anti-adhesive therapy is already in use as an alternative method to antibiotic therapy.

However, the effect of prebiotics on the adhesion of probiotics remains unclear. Previously, we have shown that the presence of prebiotics results in a general decrease in adherence in several preand probiotic combinations [8]. For this reason, the objective of this study was to examine the influence of prebiotics on the adherence of lactobacilli and thus furthering our knowledge regarding their interactions. This was conducted by testing the effect of commonly used commercial fructan-type prebiotics on the adherence of five lactobacilli strains.

\section{Method}

\section{Bacterial strains and prebiotics}

The Lactobacillus delbrueckii subsp. bulgaricus CCDM 66 and
Lactobacillus animalis CCDM 382 strains used in this study were obtained from the Culture Collection of Dairy Microorganisms (CCDM, Laktoflora, Czech Republic).Three new gastrointestinal tract isolates were tested as well; Lactobacillus casei subsp. paracasei PE1TB-P, Lactobacillus gasseri PHM-7E1 (both from biopsy samples), and Lactobacillus fermentum RL 25 (from infant faeces).

The inulin-type fructooligosaccharide prebiotics Orafti GR, Orafti P95, and Orafti Synergy (Beneo, Belgium) were used for testing. Orafti GR mainly contains chicory inulin, and Orafti P95 mainly contains oligofructose obtained by the enzymatic hydrolysis of chicory inulin. Orafti Synergy represents a combination of inulin with selected chain lengths and oligofructose. Glucose (Sigma-Aldrich, Czech Republic) was used as a monosaccharide source.

\section{Adherence assays}

Black polystyrene 96-well microtiter plates (Nalge Nunc International, Penfield, NY, USA) were used for the adherence assays. The surface of the microtiter plates were coated with a $0.5 \mathrm{mg} / \mathrm{ml}$ porcine mucin (Sigma-Aldrich, Czech Republic) and plates without mucin were also used for testing. Plates with mucin were cultivated for $24 \mathrm{~h}$ at $4^{\circ} \mathrm{C}$. Bacterial suspensions were diluted in phosphate buffered saline (PBS) subsequent to $24 \mathrm{~h}$ cultivation in de Man, Rogosa, and Sharpe (MRS) broth. The density of the suspension was adjusted to a cell concentration of McFarland No. 1. Bacteria were fluorescently stained using $5 \mu \mathrm{M}$ of SYTO 24 solution (Life Technologies Corp., Carlsbad, CA, USA) and subsequently incubated for $30 \mathrm{~min}$ at $37^{\circ} \mathrm{C}$. Stained bacterial suspensions $(100 \mu \mathrm{l})$ were pipetted into the microtiter

${ }^{*}$ Corresponding author: Krausova G, Dairy Research Institute, Ke Dvoru 12a, 16000 Prague, Czech Republic, Tel: +420 296792 111; E-mail: gabika.kunova@gmail.com

Received December 03, 2015; Accepted December 18, 2015; Published December 28, 2015

Citation: Krausova G, Hyrslova I, Jakubec M, Hynstova I (2016) In Vitro Evaluation of Prebiotics on Adherence of Lactobacilli. J Microb Biochem Technol 8: 006-008. doi:10.4172/1948-5948.1000253

Copyright: (c) 2016 Krausova G, et al. This is an open-access article distributed under the terms of the Creative Commons Attribution License, which permits unrestricted use, distribution, and reproduction in any medium, provided the original author and source are credited. 
plates (both with and without mucin), followed by the addition of $100 \mu \mathrm{l}$ of prebiotic solution. After $60 \mathrm{~min}$ incubation at $37^{\circ} \mathrm{C}$, free and weakly adherent cells were removed by three washes with $200 \mu \mathrm{l}$ PBS buffer. Next, fluorescence was measured using a Synergy 2 reader (Bio Tek Instruments, Germany) at 485/20 nm (excitation) and 530/20 nm (emission). The data presented are the results of three independent measurements.

Percentage of fluorescence was calculated as follows:

$$
X(\%)=\frac{X_{R F U-N C}}{P C-N C}
$$

where $\mathrm{X}(\%)=$ percentage conversion of the relative fluorescence units; $\mathrm{X}_{\mathrm{RFU}}=$ well fluorescence in relative fluorescence units; $\mathrm{NC}=$ Negative Control (non-specific well fluorescence); $\mathrm{PC}=$ Positive Control (bacterial fluorescence without washing).

\section{Statistical analysis}

Statistically significant differences between the groups were determined with a Student's t-test at $P<0.05$ using Statistica 10 (StatSoft, Prague, Czech Republic) and Excel (Microsoft Corp., Redmond, WA). The following null hypothesis was selected: the adherence of bacteria with prebiotics/glucose is the same as bacteria in the control group (without prebiotics/glucose).

\section{Results}

The results shown in Tables 1 and 2 indicate the percentage of adherence to untreated microtiter plates and plates coated with mucin. An increase/decrease in adherence was determined by comparison to the control (blank) grown without any prebiotics or sugars. The presence of glucose and prebiotics resulted mainly in decreased adherence. Only one strain showed an increase in adherence without the addition of mucin and in the presence of prebiotics and glucose; L. casei subsp. paracasei PE1TB-P exhibited significantly enhanced adherence in comparison with the control (without the addition of prebiotics/glucose).

Adherence to mucin is important for applied uses. Overall, the adherence to mucin-coated plates was substantially lower, except for strains RL25 and CCDM 382. The robust adherence of Lactobacillus animalis CCDM 382 to mucin is of interest in terms of testing biofilm properties. The addition of prebiotics usually results in decreased biofilm production, however, when Orafti P95 was added, the adherence capacity remained at $32 \%$. The only incidence of increased adherence to mucin-coated polystyrene in the presence of prebiotics was observed for strain Lactobacillus gasseri PHM-7E1 subsequent to the addition of the Orafti GR and Orafti P95. Interestingly, the prebiotic mixture Orafti Synergy caused a decrease in the adherence of this same strain to mucin. Similarly, the addition of glucose also resulted in decreased adherence. For all other tested strains, a significant decrease in adherence to mucin-coated plates was observed subsequent to the addition of prebiotics and glucose.

\section{Discussion}

In this study, we found that the presence of glucose and the tested prebiotics resulted mainly in decreased adherence of lactobacilli to the intestinal wall. In contrast, a significant increase in the adherence of Lactobacillus gasseri PHM-7E1 to mucin was observed subsequent to the addition of two fructan-type prebiotics (Orafti P95 and Orafti GR). This strain is an isolate from the colon of a child and is of interest for its probiotic properties and high hydrophobicity (unpublished), which may be related to its adherence ability.

Adhesion is a complex process in which both non-specific and specific mechanisms play a role [9]. Non-specific mechanisms are influenced by the hydrophobicity or hydrophilicity of bacteria as well as the surface charge of the substrate. Specific mechanisms are mediated by specific molecules such as adhesins and complementary receptors $[10,11]$. Surface adhesion is also affected by surface roughness, chemical composition, and texture [12]. The rate of adhesion also depends on $\mathrm{pH}$ and the presence of sugars, bile salts, calcium, and other compounds [13-15]. Bile salts significantly decrease adhesion, whereas the addition of calcium has a favourable effect $[13,14]$. Furthermore, it has been demonstrated that the degree of adhesion is also dependent on bacterial concentration; it is directly proportional to the number of colony forming units (CFU) [15]. Although many factors can influence adherence, there is a lack of information about how prebiotics (saccharides) can influence the adhesion process of beneficial bacteria. A limited number of studies have focused on the effect of prebiotics or single saccharides on the adhesion of beneficial bacteria. The sugars, mannose and fucose, for instance, increase the adherence of

\begin{tabular}{|c|c|c|c|c|c|}
\hline & Orafti GR & Orafti P95 & Orafti Synergy & Glucose & blank \\
\hline Lactobacillus delbrueckii subsp. bulgaricus-CCDM 66 & $45.54 \pm 1.66^{*} \downarrow$ & $50.33 \pm 2.83^{*} \downarrow$ & $43.24 \pm 3.98^{*} \downarrow$ & $48.30 \pm 2.58^{*} \downarrow$ & $69.13 \pm 4.69$ \\
\hline Lactobacillus casei subsp. paracasei-PE1TB-P & $99.12 \pm 1.02 * \uparrow$ & $95.71 \pm 6.12^{*} \uparrow$ & $97.20 \pm 3.25^{\star} \uparrow$ & $80.59 \pm 3.16^{*} \uparrow$ & $55.35 \pm 3.71$ \\
\hline Lactobacillus fermentum-RL 25 & $12.88 \pm 1.95^{*} \downarrow$ & $11.88 \pm 2.32^{*} \downarrow$ & $10.83 \pm 2,64^{*} \downarrow$ & $10.53 \pm 1.92^{*} \downarrow$ & $16.33 \pm 1.38$ \\
\hline Lactobacillus animalis-CCDM 382 & $11.48 \pm 2.57^{*} \downarrow$ & $10.37 \pm 2.74^{*} \downarrow$ & $12.19 \pm 3.32^{*} \downarrow$ & $12.35 \pm 2.10^{*} \downarrow$ & $38.54 \pm 2.74$ \\
\hline Lactobacillus gasseri - PHM-7E1 & $27.95 \pm 1.59^{*} \downarrow$ & $27.16 \pm 1.34^{*} \downarrow$ & $29.12 \pm 2.14^{*} \downarrow$ & $25.57 \pm 1.53^{*} \downarrow$ & $47.93 \pm 3.58$ \\
\hline
\end{tabular}

Prebiotics: Orafti P95, Orafti GR, Orafti Synergy, glucose; blank, a sample without the addition of prebiotics or sugar; $\downarrow$ lower adherence than that of the blank; $\uparrow$ higher adherence than that of the blank; * statistically different from the sample without prebiotics (blank). $P<0.05$.

Table 1: Percentage of adherence in untreated (without mucin) polystyrene microtiter plates.

\begin{tabular}{|c|c|c|c|c|c|}
\hline & Orafti GR & Orafti P95 & Orafti Synergy & Glucose & blank \\
\hline Lactobacillus delbrueckii subsp. bulgaricus-CCDM 66 & $2.85 \pm 0.34^{*} \downarrow$ & $1.92 \pm 0.29^{*} \downarrow$ & $1.51 \pm 0.17^{*} \downarrow$ & $3.56 \pm 0.56^{\star} \downarrow$ & $22.04 \pm 1.05$ \\
\hline Lactobacillus casei subsp. paracasei-PE1TB-P & $2.00 \pm 0.41^{*} \downarrow$ & $2.50 \pm 0.56^{*} \downarrow$ & $2.35 \pm 0.29^{*} \downarrow$ & $0.98 \pm 0.37^{*} \downarrow$ & $12.90 \pm 1.54$ \\
\hline Lactobacillus fermentum-RL 25 & $5.10 \pm 0.53^{*} \downarrow$ & $8.39 \pm 0.37^{*} \downarrow$ & $5.76 \pm 0.89^{*} \downarrow$ & $11.13 \pm 0.56^{*} \downarrow$ & $32.29 \pm 1.61$ \\
\hline Lactobacillus animalis-CCDM 382 & $26.69 \pm 0.89^{*} \downarrow$ & $35.25 \pm 0.87^{*} \downarrow$ & $25.45 \pm 0.30^{\star} \downarrow$ & $20.08 \pm 0.55^{\star} \downarrow$ & $55.87 \pm 1.34$ \\
\hline Lactobacillus gasseri-PHM-7E1 & $8.50 \pm 1.34^{*} \uparrow$ & $9.22 \pm 1.46^{*} \uparrow$ & $5.22 \pm 0.46 \downarrow$ & $4.50 \pm 0.87 \downarrow$ & $5.50 \pm 0.34$ \\
\hline
\end{tabular}

Prebiotics: Orafti P95, Orafti GR, Orafti Synergy, glucose; blank, a sample without the addition of prebiotics or sugar; $\downarrow$ lower adherence than that of the blank; $\uparrow$ higher adherence than that of the blank; * statistically different from the sample without prebiotics (blank). $P<0.05$.

Table 2: Percentage of adherence in polystyrene microtiter plates coated with mucin 
Citation: Krausova G, Hyrslova I, Jakubec M, Hynstova I (2016) In Vitro Evaluation of Prebiotics on Adherence of Lactobacilli. J Microb Biochem Technol 8: 006-008. doi:10.4172/1948-5948.1000253

Bifidobacterium bifidum MIMBb75 to Caco-2 cells [13]. Recently, Koh et al. [16] demonstrated that the oligosaccharide tagatose has a positive effect on the adhesion of Lactobacillus rhamnosus GG. Another study [3] examined the effect of several prebiotic oligosaccharides and commercial prebiotics on the adhesion of probiotic strains (Bifidobacterium longum subsp. infantis ATCC 15697 and $L$. rhamnosus GG) to HT-29 and Caco-2 cell lines. The combination of 3 '- and 6'-Sialyllactose (the two predominant human and bovine milk oligosaccharides) and 6'-Sialyllactose on its own caused an increase in the adhesion of B. longum subsp. infantis ATCC 15697. In contrast, the combination of 3'-sialyllactose and the commercial prebiotic product Orafti P95 did not enhance adhesion [3]. In a previous study [8], we demonstrated that the interactions between pro- and prebiotics are diverse and strongly strain-specific. In these in vitro experiments we have shown that the addition of the most commonly used fructanbased prebiotics resulted mainly in a decrease in the adherence of the tested strains. It seems that certain oligosaccharides are able to enhance the adhesion of probiotics; the effect on adherence is variable and strain specific, especially for commercially available prebiotic mixtures. In the next step, additional experiments are needed to simulate more precisely the gut conditions (e.g., using biological substrates). It is of importance that colon is a complex system where various bacterial species are present and their metabolic cooperation, fermentation products, or bacteria-substrate interactions and other factors can play a role. In conclusion, these results support our previous hypothesis and indicate the need for future research that will examine single combinations of pre- and probiotics individually. In addition, the strain Lactobacillus gasseri PHM-7E1 seems to be perspective from the point of adherence ability and should be used to determine new suitable symbiotic combinations.

\section{Acknowledgement}

The authors thank the Ministry of Education, Youth and Sports of the Czech Republic (COST LD 14123) for their financial support.

\section{References}

1. Laparra JM, Sanz Y (2009) Comparison of in vitro models to study bacterial adhesion to the intestinal epithelium. Lett Appl Microbiol 49: 695-701.

2. Reid G (2008) Probiotics and prebiotics - Progress and challenges. Int Dairy J 18: $969-975$
3. Kavanaugh DW, Callaghan JO, Buttó LF, Slattery H, Lane J (2013) Exposure of Bifidobacterium longum subsp. infantis to milk oligosaccharides increases adhesion to epithelial cells and induces a substantial transcriptional response. PLoS ONE 8: e67224.

4. Shoaf K, Mulvey GL, Armstrong GD, Hutkins RW (2006) Prebiotic galactooligosaccharides reduce adherence of enteropathogenic Escherichia coli to tissue culture cells. Infect Immun 7: 6920-6928.

5. Collins MD, Gibson GR (1999) Probiotics, prebiotics, and synbiotics: approaches for modulating the microbial ecology of the gut. Am J Clin Nutr 69 : 1052S-1057S

6. Lane JA, Mehra RK, Carrington SD, Hickey RM (2010) The food glycome: a source of protection against pathogen colonization in the gastrointestinal tract. Int J Food Microbiol 142: 1-13.

7. Saad N, Delattre C, Urdaci M, Schmitter JM (2013) Bressollier, "An overview of the last advances in probiotic and prebiotic field," in LWT. Food Sci Techno 50: 1-16.

8. Kadlec R, Jakubec M (2014) The effect of prebiotics on adherence of probiotics. J Dairy Sci 97: 1983-1990.

9. Del Re B, Sgorbati B, Miglioli M, Palenzona D (2000) Adhesion, autoaggregation and hydrophobicity of 13 strains of Bifidobacterium longum. Lett Appl Microbiol 31: 438-442.

10. Pelletier C, Bouley C, Cayuela C, Bouttier S, Bourlioux P, et al. (1997) BellonFontaine, "Cell surface characteristics of Lactobacillus casei subsp. casei, Lactobacillus paracasei subsp. paracasei and Lactobacillus rhamnosus strains," Appl Environ Microbiol 6: 1725-1731.

11. Kos B, SuskoviÄ J, VukoviÄ S, Simpraga M, Frece J, et al. (2003) Adhesion and aggregation ability of probiotic strain Lactobacillus acidophilus M92. J Appl Microbiol 94: 981-987.

12. Donlan RM (2002) Biofilms: microbial life on surfaces. Emerg Infect Dis 8: 881 890

13. Guglielmetti S, Tamagnini I, Minuzzo M, Arioli S, Parini C, et al. (2009) Study of the adhesion of Bifidobacterium bifidum MIMBb75 to human intestinal cell lines. Curr Microbiol 59: 167-172.

14. Marcináková M, Klingberg TD, Lauková A, Budde BB (2010) The effect of pH bile and calcium on the adhesion ability of probiotic enterococci of animal origin to the porcine jejunal epithelial cell line IPEC-J2. Anaerobe 16: 120-124.

15. Greene JD, Klaenhammer TR (1994) Factors involved in adherence of lactobacilli to human Caco-2 cells. Appl Environ Microbiol 60: 4487-4494.

16. Koh JH, Choi SH, Park SW, Choi NJ, Kim Y, et al. (2013) Synbiotic impact of tagatose on viability of Lactobacillus rhamnosus strain GG mediated by the phosphotransferase system (PTS). Food Microbiol 36: 7-13. 